\title{
OBSERVATIONS SUR LE COMPORTEMENT DES ABEILLES BUTINEUSES
}

\author{
PAR \\ J. LECOMTE \\ Station de Recherches apicoles, Bures-sur-Yvette.
}

Le comportement des Abeilles butineuses a donné lieu à un grand nombre d'observations et de publications, mais elles ne portent généralement que sur un seul aspect du butinage; bien que nombre d'entre elles soient du plus haut intérêt, il m'a semblé intéressant de poser le problème du pourquoi et du comment de l'activité de l'ensemble des butineuses d'une ruche au cours d'une période de l'année.

Le premier travail consistait en la recherche d'une méthode destinée à permettre de reconnaitre les butineuses d'une ruche donnée parmi une population de butineuses quelconques observée dans la nature.

C'est essentiellement la mise au point de cette méthode que je vais exposer ici ; mais d'autre part, en effectuant cette mise au point j'ai pu effectuer un assez grand nombre d'observations que je relaterai au fur et à mesure.

\section{Lieu d'observation}

Le but poursuivi, l'établissement d'une carte de butinage pour une colonie, rend indispensable un certain nombre de conditions.

Il faut travailler dans un territoire peu peuplé, dont les parcelles ne sont pas entourées de clôtures difficilement franchissables, avec peu de cultures de grande superficie, difficiles à prospecter sans y occasionner des dégâts.

Il faut que la densité en ruches soit normale et que la valeur mellifère et pollinifère de la flore soit satisfaisante ; il faut enfin que les conditions de climat soient régulièrement bonnes pour que les périodes d'observation puissent coïncider avec des périodes de butinage. 
Pour toutes ces raisons, j'ai porté mon choix sur un rucher dépendant de la Station Expérimentale d'Apiculture de Montfavet; ce rucher est situé à Pougnadoresse (Gard) (I). Il est composé de 25 colonies assez hétérogènes du point de vue racial, habitant des ruches modèle Dadant, disposées dans la vallée d'une petite rivière, la Tave. Cette vallée, dans la portion qui nous intéresse, est relativement étroite (900 mètres de crête à crête) et approximativement orientée est-ouest. Le fond de la Vallée est à I 40 mètres d'altitude, la ligne de crête sud, en moyenne à 230 mètres ; la crête nord à Igo mètres. I, rucher se trouvait en I956 et en I958 à 142 mètres d'altitude, au bord de la rivière. En I959 et I960, il était à quelques dizaines de mètres de son premier emplacement, à I50 mètres d'altitude.

Je n'envisagerai pas une étude de la flore de la région, me contentant de citer les plantes fleuries et d'intérêt apicole pour chaque période d'observation.

\section{PRLMIÈRE PÉRIODE : I956.}

I a période d'observation s'étend du zo juin au I3 juillet. Cette période fut chaude (température de l'air oscillant entre 20 et $30^{\circ} \mathrm{C}$ au milieu de la journée) ; certains jours, le Mistral réduisait le nombre des sorties de butineuses.

\section{Méthodes d'observations.}

L'étude du rayon d'action de l'ensemble des butineuses du rucher fut facilitée par le fait qu'il s'agissait d'abeilles italiennes ou hybrides asse $z$ faciles à distinguer des abeilles noires des ruches avoisinantes.

Par ailleurs, plusieurs essais destinés à marquer les butineuses d'une ruche donnée furent tentés.

Il est évidemment impossible de marquer au moyen de peinture appliquée individuellement une population de plusieurs dizaines de milliers de butineuses ; aussi a-t-on cherché un procédé permettant un marquage massif.

J'ai tout d'abord employé la technique de NAIRN (I953) ; cet auteur a utilisé un mélange de talc et de poudre d'aluminium, contenu dans un dispositif spécial à l'entrée de la ruche; l'Abeille en prenant son vol se saupoudre de ce mélange et peut être reconnue dans les champs.

Outre que la présence du dispositif entrâ̂ne quelques perturbations dans le vol des butineuses, le marquage n'est pas suffisamment net pour la plupart des butineuses; pour d'autres, au contraire, la dose est excessive et paraît constituer une gêne. J'ai donc renoncé à employer ce procédé.

(I) Je tiens à remercier Mme MERCIER, propricutaire à POLGNADORLSSE (Gard) pour l'aide apportée lors de nes recherches. 
Par ailleurs, j'ai expérimenté un autre moyen de marquage, celui de Muscrave (I949-I950). Ici, on ne procède plus au marquage des butineuses d'une ruche donnée, mais au marquage de celles qui visitent une zone définie. Pour cela, les fleurs sont saupoudrées avec du talc et de la fluorescéine, deux parties pour une. I,es butineuses qui visitent ces fleurs retiennent grâce à la pilosité de leur corps, suffisamment de fluorescéine pour qu'une fluorescence puisse être observée à l'œil nu. Malheureusement, cette méthode implique le sacrifice des butineuses qu'il faut immerger pendant quelques minutes dans une solution à $0, \mathrm{I}_{5} \mathrm{p}$. IOO de potasse. J'ai tenté de rechercher ainsi quelle proportion de butineuses visitait quelques ares de trèfle blanc ; mais le nombre de butineuses retrouvées à 1 'entrée des ruches, 2 sur 500 , interdit toute statistique et pour obtenir des résultats significatifs, il faudrait effectuer des prélèvements vraiment trop importants. J'ai donc en 1956 renoncé aux méthodes de marquage et me suis contenté des observations globales, compte tenu de la différence morphologique entre les abeilles de mon rucher d'observation et celles des autres ruches.

\section{Résultats.}

Au début de la période d'observation les deux ressources mellifères les plus abondantes et les plus visitées sont constituées par le châtaignier (Castanea sativa) et les Clématites (Clématis vitalba et Clematis flammula). Mais ces deux plantes ne sont point du tout réparties de la même manière; les Châtaigniers sont bien localisés en deux emplacements principaux, d'environ un hectare chacun, alors que les Clématites sont disséminées un peu partout et ne couvrent que quelques mètres carrés à quelques ares an maximum.

En corrélation sans doute avec cet état de fait, il existe des chemins de butinage très nets vers les châtaigniers tandis qu'en direction des Clématites, il n'est pas possible d'en distinguer. J'ai donné précédemment (LECOMTE, I956) une étude de ces chemins, qui sont sonvent très visibles et qui m'ont semblé conditionnés par trois facteurs, la position du trou de vol, la position des plantes fletrie's et le relief. In ce qui concerne ce dernier point, j'ai pu confirmer le fait que les accidents de terrain manifestaient des propriétés répulsives et qu'au contraire, les brèches étaient fortement attractives. I,es 30 juin, I er 2 et 3 juillet, deux chemins très inégaux existaient, se dirigeant respectivement vers deux groupes de Châtaigniiers situés le prenier à 400 mètres, le second à 800 mètres du rucher. I,e chemin se dirigeant vers le premier groupe était trois ou quatre fois plus fréquenté; mais à partir du 4 juillet, la situation s'inversa, le nombre de butineuses devint plus important le long du deuxième chemin et ceci en corrélation avec le fait que le premier groupe de Châtai- 
gnier commençait à défleurir. Jusqu'au I2 juillet les deux chemins persistent, bien que les Châtaigniers soient très peu fleuris.

Par contre, les Châtaigniers situés en dehors des zones desservies par ces chemins furent abandonnés plus vite ; ils avaient d'ailleurs, même au temps de leur pleine floraison, reçu bien moins de visites.

Un éloignement de 50 mètres des chemins et de la masse principale de la plantation, suffit déjà à provoquer ce phénomène d'une manièrł très sensible même dans le cadre d'une grossière estimation.

Au fur et à mesure que diminue le butinage sur le châtaignier, l'on assiste à une ramification des chemins de butinage; et bien que les deux chemins dont j'ai parlé restent visibles, de nombreux chemins secondaires font leur apparition, se dirigeant vers différentes petites surfaces de trèfle blanc (Trifolium repens).

En ce qui concerne les Clématites, leur butinage ne semble pas se faire à une aussi grande distance du rucher que dans le cas des Châtaigniers. Én effet, si j'ai pu repérer des Abeilles en provenance du rucher à près d'un kilomètre de distance sur les Châtaigniers, la limite en ce qui concerne les Clématites ne dépasse pas 500 mètres. Les surfaces de Clématites les plus importantes, situées à 800 mètres environ, ne recevaient aucune visite d'Abeilles bien qu'abondamment visitées par des Apides sauvages. D'autre part, il est frappant de constater la différence d'attractivité entre diffárentes plantes; le Sureau Yèble (Sambucus ebulus) n'est visité que s'il en existe une surface supérieure à quelques mètres carrés tandis qu'un pied de Lavande (Lavandula sp.) parfaitement isolé est continuellement visité par une ou plusieurs butineuses.

Par ailleurs, certaines plantes réputées d'intérêt apicole, existaient en abondance et se trouvaient bien fleuries durant la période de mes observations, cependant, aucune abeille ne visitait ces plantes. C'est en particulier le cas de la Vipérine (Echium vulgare) et du genêt d'Espagne (Spartium junceum).

DELYIÈME PÉRIODE : I958.

I a période d'observation s'étend du i 2 juin au I 7 juin. Cette période fut assez généralement chaude et ensoleillée, la température maximum de $1^{\prime}$ air oscillant entre 20 et $25^{\circ} \mathrm{C}$.

\section{Méthodes d'observation.}

Les difficultés rencontrées lors d'essai d'application de diverses méthodes de marquage en 1956 m'ont amené à penser aux possibilités offertes par les radio-isotopes. Ce type de marquage, déjà utilisé pour 
étudier le déplacement des animaux les plus divers est d'ailleurs facilité dans le cas de l'Abeille par de continuels échanges de nourriture entre les individus. NIXON et RIBPaNDS (I952) ont étudié ces échanges, en faisant ingérer à quelques ouvrières du sirop de sucre contenant du phosphore radio-actif. Ils ont ainsi mis en évidence la rapidité de la transmission entre les membres de la colonie.

D'autre part, la dose d'un microcurie par abeille nous a paru suffisante pour une reconnaissance rapide sur le terrain et il semble avantageux d'utiliser un émetteur de rayonnement gamma afin de ne pas être gêné dans la reconnaissance par les divers écrans possibles : végétaux, parois de la ruche, etc.

En collaboration avec la section d'applications de Radioéléments du Centre d'Ettudes Nucléaires de Saclay, notre choix s'est porté sur l'or I 98 dont la période de 2,68 jouts permet d'éviter les contaminations prolongées et annule pratiquement les risques d'accidents. Pour des détails plus précis l'on peut se reporter à nos notes préliminaires (Courtors et LECOMTE, I958-I959, a).

D'autre part, il a été vérifié que la dose de radioactivité reçue au cours de l'opération était très inférieure à celle susceptible de provoquer des troubles chez l'ouvrière d'Abeilles (CouRTOIS et LECOMTE, I959, $b$ ).

Un nourrisseur contenant I litre de sirop de sucre à $50 \mathrm{p}$. 100 et $5 \mathrm{ml}$ de suspension d'or colloïdal d'une activité d'environ $40 \mathrm{mc}$ fut placé à l'intérieur de la colonie le Ir juin à I 8 heures. Le I2 juin à 9 heures, sur 55 butineuses capturées au trou de vol, 35 se trouvaient marqué ss de manière nette. Le I2 à I9 heures, 45 sur 50 ; enfin, les I3, I4, I5 et I6, Ioo p. Ioo des butineuses étaient marquées de manière satisfaisante. Toutes les ressources mellifères et pollinifères furent repérées dans un rayon $\mathrm{d} \equiv 3 \mathrm{~km}$ autour de la ruche étudiée et des prélèvements d'Abeilles furent effectués sur les lieux de butinage en I 6 points différents.

I a reconnaissance des Abeilles marquées est parfois possible en approchant le détecteur de la butineuse; mais seulement si cette dernière est suffisamment immobile. Le plus souvent, il est plus avantageux de capturer l'ouvrière avec un filet à insecte et de la relâcher après l'avoir examinée.

Cette dernière méthode permet en particulier d'étudier les butineuses de la cîme des arbres et permet d'utiliser une constante de temps élevée de l'appareil détecteur, accroissant ainsi sa sensibilité et facilitant le repérage. I 027 butineuses ont pu être ainsi examinées et parmi elles I7 radioactives.

Ce pourcentage assez faible en apparence est dî au fait qu'il n'y avait qu'une ruche marquée au milieu d'un rucher de trente colonies et que huit autres colonies se trouvaient ì environ I 000 mètres de distance. 
Le tableau ci-dessous et la carte ci-jointe résument les résultats.

Un prélèvement a par ailleurs été effectué parmi les butineuses d'eau, au point marqué S sur la carte. Sur 65 butineuses, 3 furent trouvées radioactives. Enfin un certain nombre de plantes fleuries étaient visitées par des Apides sauvages et non par les Abeilles.

\begin{tabular}{|c|c|c|c|}
\hline Prélèvements & l'lantes visitées & $\begin{array}{l}\text { Abeilles } \\
\text { capturées }\end{array}$ & $\begin{array}{l}\text { Abeilles } \\
\text { marquées }\end{array}$ \\
\hline $\mathrm{I}$ & Troëne (Ligustrum vulgare) & 5 & I \\
\hline 2 & Moutarde (Sinapis arrensis) & 25 & 2 \\
\hline 3 & Moutarde (Sinapis arvensis) & 35 & I \\
\hline 4 & Châtaignier (Castanea satiz'a) & 79 & o \\
\hline 5 & Mauve (.Mala'a sy/vestris) & 9 & I \\
\hline 6 & Vesce (Vicia sativa) & ISo & o \\
\hline 7 & Veble (Sambuscus ebulus) & 48 & 2 \\
\hline 8 & Vesce (Vicia satiza) & $9 \mathrm{I}$ & 4 \\
\hline 9 & Chátaignier (Castanea rulgart) & 00 & I \\
\hline 10 & Moutarde (Sinapis arrensis) & 89 & 0 \\
\hline I 1 & Mauve (Malva syliestris) & 52 & 0 \\
\hline I 2 & Mauve (Malva sylneslris) & 44 & o \\
\hline 13 & Clénatite (Clemalis Vitalba) & 32 & 0 \\
\hline It & Mauve (Malva sylvestris) & 140 & 0 \\
\hline $\mathrm{I} 5$ & Tèble (Sambuscirs ebulus) & 25 & 2 \\
\hline 16 & Tilleul (Titia platyphyllos) & is & o \\
\hline
\end{tabular}

I, on trouve ainsi les emplacements :

$a$ : Coquelicot (Papaver Rheas) et Crepis sp.

$b$ : Coquelicot et Moutarde.

$c$ : Troène, Luzerne (Medicago sativa), Moutarde ; Clématite; Sainfoin (Onobrychis sativa).

On remarquera en lisant ce tableau, que par suite du peu de temps disponible, les emplacements où l'on rencontrait des abeilles marquées, étaient rapidement abandonnés puisque nous avions acqui : la certitude que les Abeilles de notre ruche les prospectaient. Par contre, dans les champs où aucune ouvrière marquée n'était retrouvée, nous avons, dans la mesure du possible, continué à collecter des butineuses.

On trouvera dans la conclusion générale, un essai d'interprétation de ces résultats.

TROISIÈME PÉRIODE L'OBSERVATION : I950.

En I959; un marquage similaire fut tenté le 20 mai.

Ia ruche utilisée était le siège d'une grande activité due à une forte miellée d'acacias (Robinia psendacacia). La semaine qui suivit l'opération de marquage fut caractérisée sur le plan météorologique par un ciel couvert, d'abondantes chutes de pluie et une température assez basse ( $15^{\circ} \mathrm{C}$ à $20^{\circ} \mathrm{C}$ maximum). 


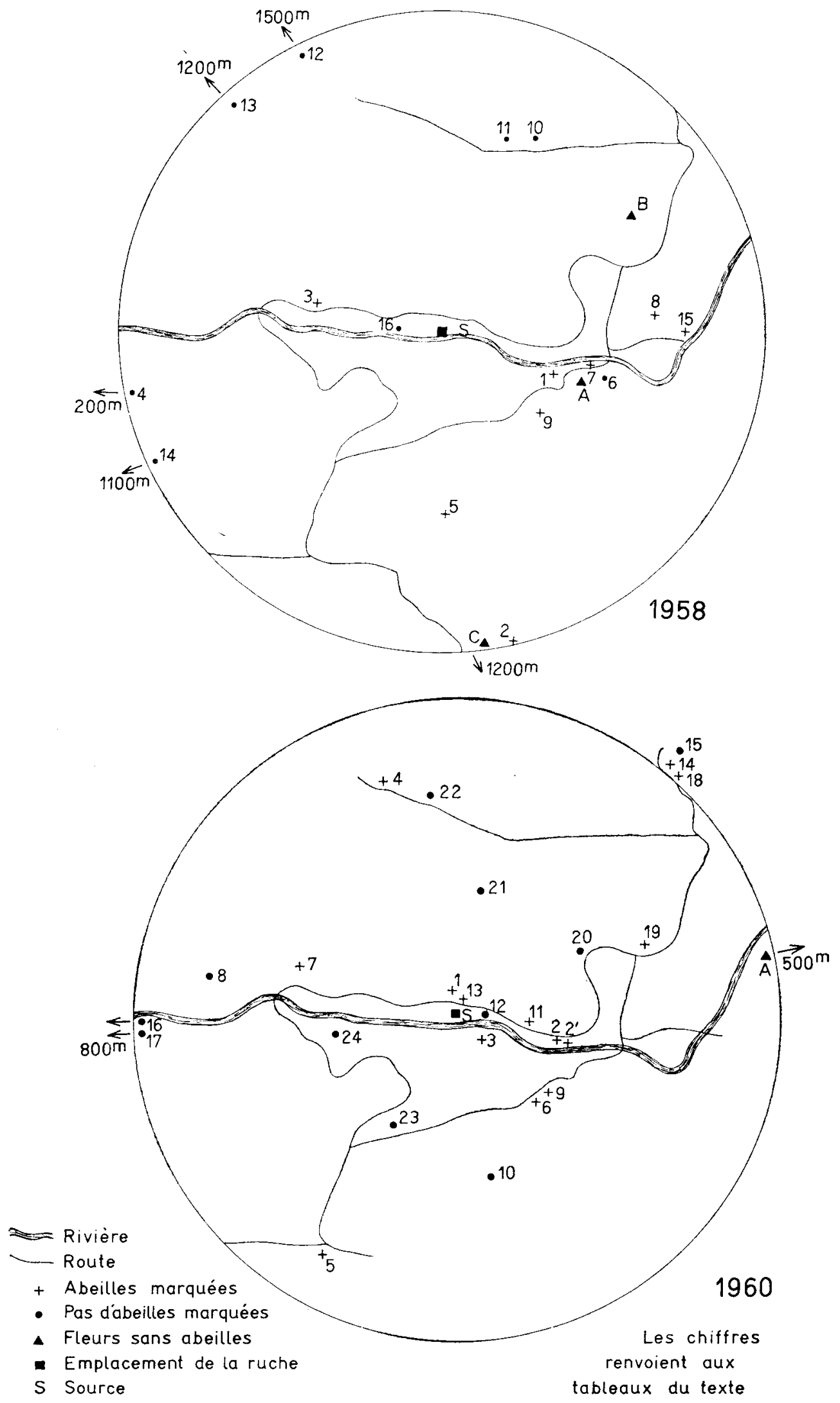


Les résultats de marquage furent notablement différents de ceux obtenus en 1958 .

En effet, bien que le sirop de sucre ait été absorbé avec plus de rapidité que dans le premier cas, les pourcentages de butineuses marquées furent loin d'être aussi importants.

Après 15 heures, I $2 \mathrm{p}$. Ioo des butineuses seulement présentaient des traces décelables de radio-activité, contre 63 p. Ioo au bout du même laps de temps en I958. Ce pourcentage atteignait $62 \mathrm{p}$. Ioo au bout de 24 heures, au lieu de $90 \mathrm{p}$. Ioo et jamais le pourcentage n'atteignit plus de 68 p. roo. De plus, la radio-activité des ouvrières considérées comme marquées était très faible et le repérage difficile. Dans ces conditions, il n'est pas étonnant que nous eussions seulement capturé une ouvrière marquée sur 696 butineuses. Cet essai est donc d'un intérêt nul en ce qui concerne l'étude du butinage, par contre, il attire notre attention sur la question des échanges de nourriture à l'intérieur de la ruche. Je me propose d'en discuter dans la conclusion de cette étude en utilisant les résultats de $\mathrm{rg60}$.

\section{QUATRIÈME， PÉRIODE : Ig60.}

La période d'observation prend place dı 5 mai au ro mai.

Sur le plan météorologique, elle est caractérisée par un temps très ensoleillé et une température assez élevée; la température de l'air variant entre $22^{\circ} \mathrm{C}$ et $26^{\circ} \mathrm{C}$ maximum.

Le marquage commencé $1 \mathrm{e} 4$. mai à I $\mathrm{h}$ I5 se généralisa très rapidement puisque $I_{5}$ heures après Ioo $\mathrm{p}$. Ioo des butineuses étaient devenues radio-actives. Cependant, l'élimination du radic-isotope fut notablement plus rapide qu'en I958 et le 7 mai à 15 h 30 , si Ioo p. Ioo des butineuses étaient encore marquées, le niveau était devenu trop faible pour assurer une reconnaissance aisée. Aussi, à 18 heures, un second marquage fut réalisé dans les mêmes conditions et permit de continuer la prospection. En 1960 , grâce à ce double marquage, 2442 ouvrières purent être capturées et $8 \mathrm{I}$ butineuses marquées purent être retrouvées.

Le tableau ci-dessous et la carte ci-jointe donnent le détail des captures.

En outre, un prélèvement effectué à l'emplacement où les butineuses se ravitaillaient en eau, a permis de capturer trois ouvrières marquées sur 79 .

Un peuplement assez important de Cistes, absolument dédaigné par les Abeilles, a été repéré et signalé sur la carte par la lettre A. 


\begin{tabular}{|c|c|c|c|c|}
\hline $\begin{array}{l}\text { Prélève. } \\
\text { ments }\end{array}$ & Plantes visitées & Importance & $\begin{array}{l}\text { Abeilles } \\
\text { capturées }\end{array}$ & $\begin{array}{l}\text { Abeilles } \\
\text { marquées }\end{array}$ \\
\hline $\mathbf{I}$ & Ciste (Cislus albidus) & épars, $50 \mathrm{~m}$ diametre & 125 & 5 \\
\hline 2 & Crepis sp. & zo ares & 100 & 33 \\
\hline $\mathbf{2}^{\prime}$ & Mélilot (Melilotus officinalis) & 20 ares & 100 & 8 \\
\hline 3 & Sainfoin (Onobryclizs sativa) & 50 ares & 212 & .3 \\
\hline 4 & $\begin{array}{l}\text { Ciste - Thym (Thymus sp.) } \\
\text { Moutarde (Synapis arvensis) }\end{array}$ & épars, roo m diamêtre & Ior & I \\
\hline 5 & Renoncule (Tramuncilus sp.) & 20 hectares & $12 \mathrm{I}$ & I \\
\hline 6 & Acacia & I 5 arbres & I 50 & I \\
\hline 7 & Ciste & épars, $200 \times 20 \mathrm{~m}$ & 108 & 7 \\
\hline 8 & Renoncule, Vesce, Moutarde & épars, $100 \mathrm{~m}$ diametre & 3 & 0 \\
\hline 9 & Ciste & $15 \mathrm{~m}^{2}$ & 100 & 10 \\
\hline ro & $\begin{array}{l}\text { Cistes (C: albidus, et Mons- } \\
\text { pelliensis) }\end{array}$ & épars, $200 \times 50 \mathrm{~m}$. & I0O & 0 \\
\hline II & Acacia & 2 arbres & 10,3 & 6 \\
\hline I2 & Acaciat & 20 arbres & 75 & 0 \\
\hline I3 & Thym & $1111^{2}$ & 56 & 2 \\
\hline I4 & Thym & épars, $100 \mathrm{~m}$ diamètre & 57 & I \\
\hline 15 & Vesce (Vicia salina) & ro ares & 122 & $\circ$ \\
\hline 16 & Sainfoin & 20 ares & 105 & o \\
\hline I 7 & Thym & épars, $50 \mathrm{~m}$ diamètre & 105 & 0 \\
\hline 18 & Ciste & épars, too m diamètre & 125 & 2 \\
\hline I9 & Ciste & $10 \mathrm{~m}^{2}$ & 49 & 3 \\
\hline 20 & Ciste & $5 \mathrm{~m}^{2}$ & 40 & 0 \\
\hline $2 \mathrm{I}$ & Ciste & 'Très dense, 20 ares & IOI & 0 \\
\hline 22 & Crepis, Mélilot, Ciste & épars, soo m diamètre & IOO & 0 \\
\hline 23 & Thym & épars, $50 \mathrm{~m}$ diametre & 29 & 0 \\
\hline 24 & Sainfoin & jo ares & I I I & 0 \\
\hline
\end{tabular}

\section{Conclusions}

Les résultats obtenus en I958 et surtout en r960 montrent qu'une étude du comportement des Abeilles butineuses peut être envisagée au moyen de la méthode de marquage à 1'or I98. Cependant, les résultats paradoxaux de I959 nous montrent le peu d'étendue de nos connaissances sur les échanges de nourriture à l'intérieur de la ruche.

La seule différence apparente entre les conditions de ces essais est le fait qu'en r959 la miellée était très abondante ; les conditions météorologiques ne devinrent mauvaises que le lendemain de la distribution du sirop, alors qu'en I958 et I960, la miellée était très faible. Dans le premier cas, elle se terminait, dans le second, elle débutait. D'ailleturs, sans qu'il soit possible de donner des chiffres précis, le deuxième marquage de rg6o effectué au moment où la miellée d'Acacia commençait à prendre de l'ampleur, a été nettement moins rapide à se généraliser que le premier.

En I959, un examen de la ruche mit en évidence que le sirop de sucre radio-actif avait été stocké dans les rayons; sans doute n'y eut-il que peu d'échanges entre les butineuses rapportant le nectar de l'extérieur et celles ayant recueilli le sirop de sucre en provenance du nourrissetur placé à l'intérieur de la ruche.

Peut-être n'est-il pas impossible d'avancer qu'en période de grande miellée, les différents groupes de butineuses travaillant sur des plantes ditférentes n'ont pas beaucoup de contacts. Ce phénomène permettrait à des 
"groupes de travail " de butiner des fleurs à parfums très différents sans qu'il puisse y avoir interférence.

Quels sont les résultats concernant le comportement de butinage obtenus lors de ces essais préliminaires destinés surtout à nous fournir une méthode de travail?

I.e rayon d'action paraît assez limité. En I958 comme en I960, aucune butineuse ne dépasse sensiblement I kilomètre de distance et le plus gros de la troupe se trouve à l'intérieur d'un cercle de $600 \mathrm{~m}$ de diamètre. Ceci confirme les vues de différents auteurs, par exemple 'Tzygankov (I953), Beuther (I954). L'hétérogénéité de la dispersion est frappante; deux champs de fleurs différents placés côte à côte, peuvent être l'un visité, l'autre non. Deux champs de la même plante situés en deux emplacements différents, peuvent, également, être ou non visités. Une intéressante observation a pu être faite en I96o à l'emplacement 2. Cet emplacement correspond à une vigne en friche où se produisait au début de la période d'observation une abondante floraison de Crepis. 'Très rapidement les Crepis disparurent et furent remplacés par du Mélilot $\left(2^{\prime}\right)$; corrélativement le pourcentage d'abeilles marquées dininua de manière sensible. Sur les Crepis, un premier prélèvement effectué le 5 mai permit de retrouver I6 ouvrières marquées sur 50 ; le 6 mai, I7 sur 5o. Le 9 mai, alors qu'il ne restait plus de Crepis, un prélèvement de roo butineuses ne permit de retrouver que 8 abeilles marquées. Si d'autres exemples venaient confirmer ces données, 1'on pourrait évaluer les valeurs respectives de l'attachement à une plante donnée et à un emplacement donné. En ce qui concerne l'attachement à un emplacement donné, j'ai pu confirmer de manière très sérieuse, les vues déjà exposées sur les facteurs qui conditionnent le choix des secteurs de butinage.

$L$ 'effet $d u$ relief est considérable; non seulement les accidents de terrain ou les barrières végétales ne sont franchies qu'avec répugnance, mais encore, immédiatement après un obstacle, les ressources mellifères ou pollinifères ne sont absolument pas exploitées. Pour employer une image, si au-dessus de la ruche, se trouvait une source lumineuse puissante, le cône d'ombre dî aux obstacles correspondrait aux zones presque complètement dédaignées par les butineuses. Cependant, en dehors de ce facteur, il doit en exister d'autres, inconnus ; par exemple, rien ne permet d'expliquer pourquoi le prélèvement I2 de Ig60 n'a pas permis de retrouver d'abeilles marquées, alors qu'on l'effectuait à proximité immédiate de la ruche, et sans obstacle. Un autre facteur important est l'effet répulsif de grandes étendues stériles : les peuplements de cistes ou de thyms isolés dans la garrigue ne sont absolument pas visités par les abeilles bien que couverts d'Apides sauvages ; de même, en I958, 1es plantes du point $C$.

Bien des points restent encore obscurs et cette étude ne doit être 
considérée que comme la mise au point d'une méthode de travail. Ëlle doit être complétée par d'autres analyses; en particulier une étude quantitative et qualitative de la sécrétion nectarifère serait souhaitable.

D'autre part, le nombre des captures devra être intensifié pour permettre une discussion statistique des résultats. Il ne faut pas oublier qu'il existe environ 35 à 40 ruches dans un rayon de 2 kilomètres autour de la colonie étudiée et que cette densité est d'ailleurs indispensable pour créer des conditions de compétition normale.

D'autre part, les conditions variant sensiblement d'un jour à l'autre en fonction de l'évolution des floraisons, il y a intérêt à effectuer les prélèvements le plus rapidement possible. Enfin, certaines plantes ne sont visitées qu'à heure fixe : les cistes, jusqu'à I3 heures, le tilleul en I959 seulement jusqu'à 8 heures. Tout ceci, joint au fait qu'à part de rares exceptions une équipe de trois prospecteurs n'arrive pas à examiner plus de Ioo butineuses à l'heure, souvent beaucoup moins, et sans tenir compte des déplacements, rend nécessaire un grand effort si l'on veut établir des cartes de butinage valables.

\section{RÉFÉRENCES BIBLIOGRAPHIQUES}

Beutler (R.). - - Ueber die Flugweite der Bienen. Z. Vergl. Physiol., 36, p. 266-298, I954.

Courtors (G.) et Lecomte (J.). - Sur un procédé de marquage des Abeilles butineuses au moyen d'un radio-isotope. C. R. Acad. Sci., 24\%, p. I47I49, I958.

Courtors (G.) et LECOMTE (J.). - Marquage d'abeilles au moyen d'or radioactif. Int. J. of App. Rad. and Isotopes. Vol. 5, p. 265-268, I958 a.

Courtors (G.) et LEComTe (J.). - Sur la résistance au rayonnement gamma de $1^{\prime}$ Abeille ouvrière. Annales de l'Abeille, No 4, p. 285-290, I959 $a$.

Musgrave (A. J.). - - The use of a fluorescent material for marking and detecting. Canad. Entomologist. Vol. I,XXXI, 7, p. I73, I949.

Musgrave (A. J.). - A note on the dusting of crops with fluorescein to mark visiting. Canad. Entomologist., Vol. LXXXII, 9, p. I95, I950.

NAIRN (R. G.). - A new method of marking bees. N. Z. J. Agric., Vol. 86, 6, p. 529, I953.

Nixon (H. L.) et Ribbands (C. R.). - Food transmission in the honeybee community. Proc. Roy. Soc., B. I40, p. 43-50, I952.

Tzygankov (S. K.). - Pollinisation par les abeilles de la luzerne destinée aux semences (en russe). Pchelovodstvo, 6, p. 33-36, I953. 